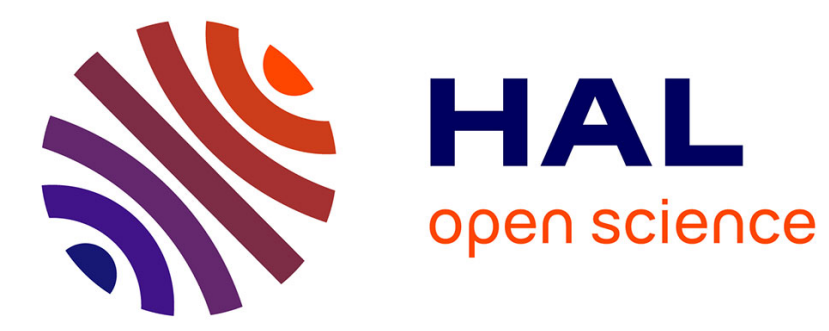

\title{
The damping of forced librations of triaxial satellites with eccentric orbits: Consequences on the dynamics of Mimas
}

Gérard Caudal

\section{- To cite this version:}

Gérard Caudal. The damping of forced librations of triaxial satellites with eccentric orbits: Consequences on the dynamics of Mimas. Icarus, 2017, 286, pp.280-288. 10.1016/j.icarus.2016.10.011 . insu-01386420

\section{HAL Id: insu-01386420 \\ https://hal-insu.archives-ouvertes.fr/insu-01386420}

Submitted on 7 Nov 2016

HAL is a multi-disciplinary open access archive for the deposit and dissemination of scientific research documents, whether they are published or not. The documents may come from teaching and research institutions in France or abroad, or from public or private research centers.
L'archive ouverte pluridisciplinaire HAL, est destinée au dépôt et à la diffusion de documents scientifiques de niveau recherche, publiés ou non, émanant des établissements d'enseignement et de recherche français ou étrangers, des laboratoires publics ou privés. 


\section{The damping of forced librations of triaxial satellites with eccentric orbits: consequences on the dynamics of Mimas \\ Gérard CAUDAL}

\section{LATMOS/IPSL, UVSQ Université Paris-Saclay, UPMC Univ. Paris 06, CNRS, Guyancourt,} France

\section{Abstract}

Saturn's moon Mimas is a triaxial body orbiting close to the planet on an eccentric orbit, and as a consequence it is librating significantly. Libration is usually believed to enhance dissipation within a planetary satellite. In this paper Mimas' poorly understood strong inward migration obtained by Lainey et al. (2012a) is interpreted as an effect of dissipation within the librating moon. Tajeddine et al. (2014) performed observations of the phase and amplitude of libration and proposed several interior models, from which they retained only two models compatible with observations: a solid body with nonhydrostatic core, or a 3-layer body including an inner dense core, surrounded by a water ocean and an icy solid shell. In this paper I combined three major observations: libration amplitude and libration phase observed by Tajeddine et al. (2014), and inward migration da/dt obtained by Lainey et al. (2012a). A further study by Lainey et al. (2015) however tends to indicate that the Mimas' strong inward motion might be questioned, and therefore this paper also explores situations with smaller $\mathrm{da} / \mathrm{dt}$. Within the assumption that inward migration is mainly due to librational dissipation, the solid model with nonhydrostatic core is found to be inconsistent with the observations. In contrast, a 3-layer model including core, ocean and shell is compatible. The observations permit to determine the icy shell depth $\mathrm{h}$, core equatorial flattening $\beta_{\mathrm{i}}$ and core pendulum quality factor $\mathrm{Q}_{\mathrm{Pei}}$, provided that an assumption is made concerning the core density. Due to the uncertainty of da/dt inferred from observations, the value of $\mathrm{Q}_{P e i}$ is however rather uncertain. Dissipation within the oceanic boundary layers is found to contribute significantly to the total dissipated power.

\section{Introduction}

Mimas, the innermost mid-sized icy moon of Saturn, has several orbital and physical intriguing peculiarities. Unlike the other mid-sized Saturn's icy moons (Enceladus, Tethys, Dione and Rhea), it has a significant eccentricity ( $\approx \approx 0.02)$. As concerns its shape, it may be modeled as a triaxial ellipsoid with significant deviation from axisymmetry. For a satellite close to the planet, such a triaxiality, combined with orbital eccentricity, are known to produce significant azimuthal libration superimposed on the synchronous rotation. The libration amplitude measured by Tajeddine et al. (2014) was further found to be about twice the libration amplitude expected from the observed eccentricity and triaxiality assuming an homogeneous body. This led those authors to propose more complex inhomogeneous interior models for Mimas.

As concerns the orbital properties, an unexpected large acceleration of Mimas, related to a secular decrease of its semi-major axis ( $\left.\mathrm{da} / \mathrm{dt} \approx-15.7 \times 10^{-15} \mathrm{AU} / \mathrm{day}\right)$, was found by Lainey et al. (2012a) when fitting a numerical orbital model of the Saturnian moons to astrometric data spanning more than a century. The combination of such observations calls to explore the possible link between libration and secular inward migration of a planetary satellite. 
The spin-orbit behavior of a satellite with a triaxial torque has been studied by several authors (Wisdom et al., 1984; Murray and Dermott, 1999; Makarov and Efroimsky, 2014; Makarov et al., 2016). Makarov and Efroimsky (2014) and Makarov et al. (2016) stressed the key role played by the physical librations in the tidal heating process. They pointed out that the planet's triaxiality should be a significant factor determining the dissipation rate at spin-orbit resonances. Tiscareno et al. (2009) expressed the damping of free libration in terms of a quality factor of the satellite. However, the orbital consequences of the dissipation of driven libration have not been much discussed in the literature. Here the effect of the triaxial torque is presented and the dissipation rate within the satellite is discussed. Among the various planetary moons of the solar system, those whose orbits are the most affected by librational dissipation are those filling several conditions, namely: irregular shape, significant eccentricity, and closeness to the planet. Mimas is particularly concerned by this effect.

Section 2 presents the main physical bases concerning the damping of driven libration of triaxial satellites. Section 3 clarifies the definitions of quality factors of the librating satellite. In section 4 the consequences on the dynamics and possible interior model of Mimas are deduced.

\section{Basic equations for forced librations}

Here we consider the motion of a planetary satellite whose spin axis is normal to the plane of its fixed elliptical orbit with eccentricity $\mathrm{e}<<1$. The satellite is assumed to be in synchronous rotation, and the masses of the satellite and the planet are $\mathrm{m}_{\mathrm{S}}$ and $\mathrm{M}_{\mathrm{P}}$, respectively, with $\mathrm{m}_{\mathrm{S}}<<\mathrm{M}_{\mathrm{P}}$. Under the effect of eccentricity, the satellite will undergo forced librations. As discussed by Makarov et al. (2016), the amplitude of the eccentricity driven libration is defined by a combination of two different types of torques: a tidal torque and a triaxial torque. The tidal torque is due to the occurrence of a tidal bulge which interacts with the planetary potential. In contrast, the triaxial torque is due to the interaction of the permanent bulge with the planetary potential. In this section, we will discuss about the rate of energy dissipation within the moon's interior by these torques.

\subsection{Dynamical equations in the undamped case:}

For a Keplerian orbit, it is well known that, to lowest order in e, the line drawn from the satellite to the empty focus of the Keplerian ellipse rotates with uniform angular frequency n, equal to the mean motion (Murray an Dermott, 1999, page 44). Since we assume that the satellite is synchronously rotating, in a classical approach, one uses a reference frame that is centered on the satellite and rotates with the satellite's mean motion $\mathrm{n}$. In this rotating frame the planet moves about its guiding centre in a 2:1 ellipse as shown in Figure 1. As a consequence, the torque exerted by the planet on the satellite permanent bulge will produce librations around the position of stationary equilibrium, which is also the line connecting the satellite to the empty focus, and is labeled as line F'F in Figure 1.

As seen in Figure 1, in this satellite centered rotating frame, we introduce the following angles: $\varphi=2$ esin(nt) between the directions of the planet and of the empty focus, $\psi$ between 
the moon's long axis and the direction of the planet (optical libration), and $\gamma=\varphi-\psi$ between the moon's long axis and the direction of the empty focus (physical libration).

Expressing the torque exerted by the planet on the permanent bulge of the moon, but ignoring the torque on the tidal bulge (which is 0 in the undamped case), one obtains the following equation of motion for $\gamma$ (Murray and Dermott, 1999, page 201):

$$
C \ddot{\gamma}-\frac{3}{2}(B-A) \frac{G M_{P}}{r^{3}} \sin 2 \psi=0
$$

where $\mathrm{A}<\mathrm{B}<\mathrm{C}$ are the three principal moments of inertia of the moon, $\mathrm{C}$ being the moment about the spin axis, $\mathrm{G}=6.67 \times 10^{-11} \mathrm{~m}^{3} \mathrm{~kg}^{-1} \mathrm{~s}^{-2}$ is the gravitational constant, and $\mathrm{r}$ is the moonplanet distance. Since the semi-major axis a of the satellite is related to mean motion $n$ through $\mathrm{GM}_{\mathrm{P}} / \mathrm{a}^{3}=\mathrm{n}^{2}$, equation (1) can be rewritten, to lowest order of $\mathrm{e}$ and for small libration angle $\psi$ (Murray an Dermott, 1999, page 216):

$$
\ddot{\gamma}+\omega_{o}^{2} \gamma=2 \omega_{o}^{2} e \sin (n t)
$$

where

$$
\omega_{o}^{2}=3 \sigma n^{2}
$$

and $\sigma$ is the triaxiality factor:

$$
\sigma=\frac{B-A}{C}
$$

Note that equation (2) takes into account only the leading frequency of libration, while a more general theory with all the frequencies involved is provided by Comstock and Bills (2003). Equation (2) describes a forced harmonic oscillator, whose well known solution is the forced libration:

$$
\gamma=\frac{2 e \sin (n t)}{\left.1-\frac{1}{3 \sigma}\right)}
$$

One sees that, in the case where $\sigma<1 / 3, \gamma$ oscillates in phase opposition with $\varphi$. In all cases, since $2 \operatorname{esin}(\mathrm{nt})=\varphi=\gamma+\psi$ (as seen above), equation (5) implies $\gamma=-3 \sigma \psi$.

\subsection{The effect of tidal damping in the case of very small triaxiality $(\sigma<<1)$ :}

Most moons are close enough to spherical that $\sigma<<1$, and thus from equation (5) the physical libration $\gamma$ approaches zero. This means that most moons remain close to synchronous rotation, with the long axis always pointing toward the empty focus. 
Within such satellites, the energy dissipation rate due to the tidal torque as a function of eccentricity can be computed (Peale and Cassen, 1978; Yoder and Peale, 1981; Efroimsky and Makarov, 2014; Makarov and Efroimsky, 2014). To lowest orders in eccentricity, provided that the obliquity is set to zero, it may be written as:

$$
\langle-\dot{E}\rangle_{T I D E}=\frac{21}{2} \frac{k_{2 S}}{Q_{S}} \frac{G M_{P}^{2} R_{S}^{5}}{a^{6}} n e^{2}
$$

where $R_{S}$ is the satellite's radius, $Q_{S}$ its tidal quality factor and $k_{2 S}$ its Love number, which may include a correcting factor in the case of a partially molten interior.

A proportion $3 / 7$ of the dissipation of equation (6) is due to the radial tide, while $4 / 7$ of it is due to the librational tide (Murray and Dermott, 1999, p. 172). Since there is virtually no physical libration here $(\gamma \approx 0)$, librational dissipation is due to the oscillation of angle $\psi$ of Figure (1) ("optical libration").

Since the total orbital energy of the planet-satellite system is $E=-G m_{S} M_{P} / 2 a$, and the rotational energy associated with the spin of the corotating moon is negligible compared to the orbital energy, the secular inward motion of the satellite can be written:

$$
\dot{a}=-(a / E)\langle\dot{E}\rangle
$$

\section{(7)}

and thus equation (6) may be expressed in terms of the inward motion of the satellite due to the tidal effect:

$$
\dot{a}_{\text {TIDE }}=-21 \frac{k_{2 S}}{Q_{S}} \frac{R_{S}^{5}}{a^{4}} \frac{M_{P}}{m_{S}} n e^{2}
$$

\subsection{The effect of damping for triaxial satellites $(\sigma \neq 0)$ :}

As argued by Makarov and Efroimsky (2014), physical libration plays a key role in the tidal heating process of a close-in planetary satellite. The amplitude of physical libration being dependent upon triaxiality in equation (5), a body with a more pronounced triaxiality should generate significantly more dissipation than a similar body of a more symmetrical shape. The first direct cause of dissipation is the classical phase lag of the tidal bulge of the librating satellite with respect to the planetary forcing. In addition, the physical librations described in section 2.1 will produce horizontal alternating inertial forces within the moon. Yoder (1982) discussed the question of the induced toroidal distortion in the context of Phobos, described as an incompressible homogeneous sphere. Moreover, in contrast to most fluids and monocrystalline solids, earth materials exhibit nonlinear elastic behavior, as detailed in the review by Ostrovsky and Johnson (2001). The presence of soft small features referred as "bond system" (microcracks, grain contacts, dislocations, ...) within a hard matrix (grains, crystals) produces a nonlinear response, characterized by hysteresis and slow dynamical 
effects (relaxation) at microscale. In presence of the azimuthal alternating acceleration due to libration, the hysteretic response of the microscale bond system is expected to also contribute to dissipation, which will add to the dissipation produced by macroscopic (radial or toroidal) distortions of the body. In other words, when forced by the longitudinal physical libration, the material within the moon will respond with a certain delay, associated with a dissipation which is not related to the presence of a tidal bulge.

Rather than detailing those complex processes involved in dissipation (radial and toroidal distortions, microscale hysteresis), I follow the approach by Tiscareno et al. (2009) who include them through a unique damping term in equation (2). Although nonlinearity is certainly present in the system, the lowest order approach is to account for all the damping processes through a linear damping term $b \dot{\gamma}$ in equation (2), yielding the driven damped harmonic oscillator equation:

$$
\ddot{\gamma}+b \dot{\gamma}+\omega_{o}^{2} \gamma=2 \omega_{o}^{2} e \sin (n t)
$$

where $\mathrm{b}$ is an unknown friction parameter. Following previous authors (Murray and Dermott, 1999; Tiscareno et al., 2009), one may relate parameter b to the quality factor $Q$ of the oscillating satellite, by first considering the free oscillator, obtained by removing the forcing term $2 \omega_{o}{ }^{2} e \sin (n t)$ from the right hand side of equation (9), and applying the definition of the quality factor :

$Q_{P e}=\frac{2 \pi E_{o}}{\Delta E}$

where the suffix Pe is used to recall that $\mathrm{Q}_{\mathrm{Pe}}$ is the quality factor of the pendulum described by equation (9). $\Delta \mathrm{E}$ is the energy dissipated over one cycle, and $\mathrm{E}_{\mathrm{o}}$ is the peak energy stored by the oscillator during the cycle. One gets (Tiscareno et al., 2009) :

$$
\begin{aligned}
& b=\omega_{o}\left[2-2 \sqrt{1-\frac{1}{Q_{P e}^{2}}}\right]^{1 / 2} \\
& \approx \frac{\omega_{o}}{Q_{P e}} \text { for } Q_{P e}>>1
\end{aligned}
$$

In practice, this approximation may be adopted as soon as $\mathrm{Q}_{\mathrm{Pe}} \geq 2$. This will be done henceforth in the paper.

By substituting the trial complex solution $\bar{\gamma}=\bar{\gamma}_{o} \operatorname{exp(int)}$ in equation (9), one can obtain the amplitude of the steady state response of the system: 
$\gamma_{o}=\left|\bar{\gamma}_{o}\right|=\frac{2 \omega_{o}^{2} e}{\left[\left(\omega_{o}^{2}-n^{2}\right)^{2}+n^{2} b^{2}\right]^{1 / 2}}$

this may be also written as a function of $\sigma$ and $\mathrm{Q}_{\mathrm{Pe}}$ :

$$
\gamma_{o}=\frac{2 e}{\left[\left(1-\frac{1}{3 \sigma}\right)^{2}+\frac{1}{3 \sigma Q_{P e}^{2}}\right]^{1 / 2}}
$$

Similarly, the phase shift with respect to the driving torque is given by:

$$
\tan (\delta \theta)=\frac{n b}{n^{2}-\omega_{o}^{2}}
$$

Classically for the driven damped oscillator, $\delta \theta=-\pi / 2$ at resonance $\left(n=\omega_{0}\right)$, and away from resonance one has $-\pi \leq \delta \theta \leq 0$, so that the oscillator always lags the driving torque. Equation (14) may be rewritten:

$$
\tan (\delta \theta)=\frac{\sqrt{3 \sigma}}{Q_{P e}(1-3 \sigma)}
$$

if one assumes that $Q_{P e}>\sqrt{3 \sigma} /|3 \sigma-1|$ in equation (13), one retrieves an expression consistent with equation (5) valid for the undamped case:

$$
\gamma_{o} \approx \frac{2 e}{|1-1 / 3 \sigma|}
$$

The work done by the drag force over a displacement $\mathrm{d} \gamma$ in a time $\mathrm{dt}$ is $C b \dot{\gamma} d \gamma$, therefore the instantaneous rate of dissipation is $-\dot{E}=C b \dot{\gamma}^{2} \approx C \omega_{o} \dot{\gamma}^{2} / Q_{P e}$, and thus the mean rate of energy dissipation is

$$
\langle-\dot{E}\rangle=\frac{1}{2} \frac{C \omega_{o}}{Q_{P e}}\left(n \gamma_{o}\right)^{2}
$$

Inserting the value of $\gamma_{o}$ from equations (13), equation (17) may be transformed to: 
$\langle-\dot{E}\rangle=\frac{2 C \sqrt{3 \sigma} e^{2} n^{3}}{Q_{P e}\left[\left(1-\frac{1}{3 \sigma}\right)^{2}+\frac{1}{3 \sigma Q_{P e}^{2}}\right]}$

For $Q_{P e}>>\sqrt{3 \sigma} /|3 \sigma-1|$, this may be approximated as:

$$
\langle-\dot{E}\rangle \approx \frac{4}{5} \frac{m_{S} R_{S}^{2} e^{2} n^{3}}{Q_{P e}} \frac{\sqrt{3 \sigma}}{\left(1-\frac{1}{3 \sigma}\right)^{2}}
$$

where the inertial momentum $\mathrm{C}$ has been taken as $\mathrm{C} \approx(2 / 5) \mathrm{m}_{\mathrm{S}} \mathrm{R}_{\mathrm{S}}{ }^{2}$.

From equation (7) with orbital energy $E=-m_{S} a^{2} n^{2} / 2$, equation (19) can also be expressed in terms of the inward motion of the satellite, yielding:

$$
\dot{a} \approx-\frac{8}{5} \frac{R_{S}^{2} n e^{2}}{a Q_{P e}} \frac{\sqrt{3 \sigma}}{\left(1-\frac{1}{3 \sigma}\right)^{2}}
$$

\section{Pendulum quality factor $Q_{P e}$ and moon's specific dissipation $Q_{S}$ :}

\subsection{The Phobos test:}

Tiscareno et al.(2009) identified the pendulum quality factor $\mathrm{Q}_{\mathrm{Pe}}$ defined above with the satellite's quality factor Qs. Dealing with Saturn's icy satellites Janus and Epimetheus this led them to use $\mathrm{Q}_{\mathrm{Pe}} \approx 100$ assuming the satellite is composed of monolithic ice, or $\mathrm{Q}_{\mathrm{Pe}} \approx 10$ assuming rubble pile ice. I myself followed the same approach in my paper on that topic (Caudal, 2013). I must admit however that our approach was incorrect, as can be illustrated by the following paradoxical result obtained for Mars' satellite Phobos, for which precise measurements of $\dot{a}$ have been performed.

Given the mean density of Phobos, one can safely say that it is either a rubble-pile or, at the very least, a very porous and cracked body. Hence its tidal Q is of the order of 10 to 100 . Let us use $\mathrm{Q} \approx 100$ as a very conservative, probably overestimated value. The other useful parameters for the Mars-Phobos system are given in Table 1. From those parameters, equation (20) applied to Phobos with $\mathrm{Q}_{\mathrm{Pe}}=100$ yields $\dot{a} \approx-8.8 \mathrm{~cm} /$ year. As a comparison, Phobos' observed inward drift obtained from Table $\mathbf{1}$ is only 
$\dot{a}=-(2 a / 3 n) \dot{n}=-3.85 \mathrm{~cm} /$ year , and furthermore most of this inward drift is believed to be due to tides within Mars (Bills et al., 2005; Lainey et al., 2007; Jacobson, 2010).

Thus the observed moderate inward drift of Phobos $(-3.85 \mathrm{~cm} /$ year) is inconsistent with equation (20) if the pendulum quality factor $\mathrm{Q}_{\mathrm{Pe}}$ is taken as equal to the satellite's tidal quality factor $Q_{s}$. Of course the discrepancy would be even stronger if $Q_{S}=10$ were taken. The reason is that $\mathrm{Q}_{\mathrm{Pe}}$ and $\mathrm{Q}_{\mathrm{S}}$ represent indeed different quantities.

\subsection{Quality factor of a compound pendulum:}

To clarify the meaning of the pendulum quality factor $\mathrm{Q}_{\mathrm{Pe}}$ introduced above in section 2.3, let us use again the satellite centered frame rotating with the satellite's mean motion $n$. In this rotating frame, we have seen that the triaxial satellite is oscillating around the position of stationary equilibrium labeled as line F'F in Figure 1, as a result from equation (9). The reason for this oscillation is the interaction of the moon's permanent quadrupole moment with the planetary gravity potential. Such oscillation is analogous to the oscillation of a classical compound pendulum oscillating in the terrestrial gravity field. The $\mathrm{Q}$ factor of a compound pendulum is classically defined in a similar fashion by equation (10), where $E_{o}$ is the potential energy stored by the pendulum at highest elongation (also equal to the kinetic energy at zero elongation). For a terrestrial pendulum the energy $\Delta \mathrm{E}$ dissipated over one cycle is mostly due to air friction, and to a lesser extent to friction at the pivot. If in addition the compound pendulum were not a rigid body (for example if it were a body composed of a viscoelastic material), additional dissipation would result from inertial inelastic distortions of the body. This is usually however negligible compared to air and pivot friction.

If now our oscillating satellite is regarded as a compound pendulum, air and pivot friction vanish, and the sole remaining source of dissipation is due to tidal and inertial distortions within the body The compound pendulum is damped because the moon responds sluggishly to the external forcing by the planet. The quality factor $\mathrm{Q}_{\mathrm{Pe}}$ defined above is the quality factor of the pendulum. In that sense the quantity $E_{o}$ in equation (10) is the potential energy stored in the pendulum at highest elongation.

3.3. Relation between $Q_{p e}$ and $Q_{\underline{S}}$ :

The tidal specific dissipation $\mathrm{Q}_{S}$ of a satellite is defined classically as

$$
Q_{S}=\frac{2 \pi E_{o}^{\prime}}{\Delta E}
$$

where the energy $\mathrm{E}_{\mathrm{o}}{ }_{\mathrm{o}}$ is the maximal energy stored in the tidal distortion of the body (e. g., Goldreich and Soter,1966). The peak elastic energy stored in the tidal bulge, including radial and librational component is (Murray and Dermott, 1999, p. 172):

$$
E_{o}^{\prime}=\frac{63 e^{2}}{4 \tilde{\mu}_{S}}\left(\frac{R_{S}}{a}\right)^{5} \frac{G M_{P}^{2}}{a}
$$


where $\tilde{\mu}_{S}$ is effective rigidity of the satellite (ratio of elastic and gravitational forces acting at the surface). For homogeneous solid body the tidal Love number $\mathrm{k}_{2}$ is related to $\tilde{\mu}$ through $k_{2}=(3 / 2) /(1+\tilde{\mu})$. For an icy or rocky satellite, $\tilde{\mu}_{s}>>1$, thus the energy stored in the tidal deformation is mostly elastic and the gravitational contribution may be neglected, and thus $k_{2 S} \approx 3 /\left(2 \tilde{\mu}_{S}\right)$.

Conversely, the peak energy of the pendulum is (Tiscareno et al., 2009):

$$
E_{o}=\frac{1}{2} C \omega_{o}^{2} \psi_{o}^{2}
$$

where $\psi_{\mathrm{o}}=\gamma_{\mathrm{o}} /(3 \sigma)$ is the peak value of angle $\psi$ defined in section 2.1.

The relation between $\mathrm{Q}_{\mathrm{Pe}}$ and $\mathrm{Q}_{\mathrm{S}}$ may be deduced from equations (10) and (21). One finds (for $\tilde{\mu}_{S}>>1$ ):

$$
Q_{P e}=\frac{E_{o}}{E_{o}^{\prime}} Q_{S}=\frac{8}{315}\left(\frac{a}{R_{S}}\right)^{3}\left(\frac{m_{S}}{M_{P}}\right) \frac{1}{\sigma\left(1-\frac{1}{3 \sigma}\right)^{2}} \frac{Q_{S}}{k_{2 S}}
$$

For Phobos, with the data of Table 1, this gives $\mathrm{Q}_{\mathrm{Pe}}=0.75 \mathrm{Q}_{\mathrm{S}} / \mathrm{k}_{2 \mathrm{~S}}$.

The question of the Love number of Phobos has been discussed by Le Maistre et al. (2013) who estimated the range of possible values of $\mathrm{k}_{2 \mathrm{~S}}$, yielding an upper bound value of $\mathrm{k}_{2 \mathrm{~S}}=5.3 \times 10^{-4}$ for their least rigid rubble pile model.

As discussed above in section 2.3, a librating triaxial satellite does not experience only the tidal distortion depicted by the elastic energy of equation (22), but also a toroidal contribution and microscale effects resulting from inertial distortion of the librating body. The satellite thus stores an additional elastic energy. To include the poorly known effects of toroidal distortion and microscale effects, the Love number $\mathrm{k}_{2 \mathrm{~S}}$ in equation (24) should be replaced by a modified Love number $\mathrm{k}_{2}{ }_{2}>\mathrm{k}_{2}$. It is beyond the scope of this paper to discuss the precise value of $\mathrm{k}_{2 \mathrm{~S}}$. I just point out here that, given that $\mathrm{k}_{2 \mathrm{~S}} \leq 5.3 \times 10^{-4}$ for Phobos, even though $\mathrm{k}_{2}$ is expected to be significantly higher than $\mathrm{k}_{2}$, it is likely to be still much lower than 1 , so that the pendulum quality factor $\mathrm{Q}_{\mathrm{Pe}}$ defined in equation (10) must not be identified with the satellite's $\mathrm{Q}_{\mathrm{S}}$ factor. Henceforth in the paper I will express the results in terms of $\mathrm{Q}_{\mathrm{Pe}}$ rather than $\mathrm{Q}_{\mathrm{S}}$, thus avoiding the use of Love numbers, since it is $\mathrm{Q}_{\mathrm{Pe}}$ that determines the librational phase shift $\delta \theta$ (see equation (15)), which is an observed quantity of major importance in this paper.

\section{Application to Mimas:}

The evolution of the orbits of the main moons of Saturn have been studied by Lainey et al. (2012a), using astrometric data spanning more than a century. This permitted them to revisit the tidal dissipation within Saturn, and to obtain a dissipation ratio for Saturn $\mathrm{k}_{2} / \mathrm{Q}=(2.3 \pm 0.7) \times 10^{-4}$, which is about 10 times higher than previous estimates. When 
performing their fitting, they also needed to include an additional constant rate $\mathrm{da} / \mathrm{dt}$ on Mimas' semi-major axis to conform to the data. The fitted value for the additional migration of Mimas ( $\mathrm{da} / \mathrm{dt}=-(15.7 \pm 4.4) \times 10^{-15}$ AU/day) was found unexpectedly high, and the authors mentioned that such orbital decay could have significant implications on Saturn's rings.

Resonances between close external satellites and ring particles located at specific radial distances named Linblad resonances, produce spiral density waves in the rings, leading to torques on the satellites (Goldreich and Tremaine,1980). However, for an external satellite, the migration due to Lindblad resonance would be directed outward, while instead the radial migration of Mimas obtained by Lainey et al. (2012a) is inward. Another resonant ringsatellite interaction would occur if strong radial density gradients are present at the corotation resonance radius (Goldreich and Tremaine, 1979; Molnar and Dunn, 1995), but evidence of density gradient of the required strength still needs to be found. Thus, for the time being, no clearly identified process related to Saturn's rings is able to provide a convincing explanation for such a strong observed inward migration of Mimas.

More recently, Lainey et al. (2015) performed a new fit of their model, incorporating an additional set of Cassini astrometric data. When performing this new fit, they did not need to introduce the large negative da/dt that was required in Lainey et al. (2012a). These ambivalent results tend to show that Mimas' additional inward migration da/dt should be considered as a poorly constrained quantity. In order to study the sensitivity of my results to da/dt, I therefore also tested a situation in which Mimas' migration is a factor of 10 smaller than the one inferred by Lainey et al.(2012a). In that situation the additional inward migration would be of the same order as the outward migration due to dissipation within Saturn, and thus the net radial migration would virtually vanish.

If Mimas' physical libration is ignored, inward migration due to tidal dissipation within Mimas is given by equation (8). Tidal Love number $\mathrm{k}_{2}$ is related to effective rigidity $\tilde{\mu}$ through $\mathrm{k}_{2}=(3 / 2) /(1+\tilde{\mu})$. Taking $\tilde{\mu} \approx 2700$ and $\mathrm{Q}_{\mathrm{s}} \approx 100$ (Murray and Dermott, 1999, p. 173), and other parameters for Mimas given by Table 2 , equation (8) yields $\mathrm{da} / \mathrm{dt} \approx-8.3 \times 10^{-18}$ AU/day. This is less than 3 orders of magnitude below the rate of inward migration $\mathrm{da} / \mathrm{dt}=-$ $15.7 \times 10^{-15}$ AU/day obtained by Lainey et al. (2012a). Therefore the role of tidal dissipation within Mimas can be regarded as negligible if libration is ignored.

Mimas is however a triaxial body on an eccentric orbit. In this section we propose to interpret the observed inward motion of Mimas as a consequence of librational dissipation within Mimas.

Tajeddine et al. (2014) have reported observations of Mimas' librations from which they deduced constraints on Mimas' interior. From the observed libration amplitude, after considering five interior models, they retained only two possible interiors: one model consisting in a nonhydrostatic dense core surrounded by a less dense icy layer, and a 3-layer model including a dense core, a water ocean, and an icy shell. Both types of models will be addressed in this section.

\subsection{Model with nonhydrostatic core and solid shell}

In the calculations by Lainey et al. (2012a), interactions between satellites as well as the effects of tides within Saturn have been already taken into account. The only process that they 
do not address in their computation is dissipation within the satellites (excluding Enceladus). Mimas' inward secular motion da/dt that they determine (see Table 2) is defined as a supplementary effect which needs to be added in order to fit the data. Therefore we must not include dissipation within Saturn when interpreting the da/dt of Table 2 since it has already been accounted for. In this section we intend to interpret da/dt of Table 2 as an effect of librational dissipations within Mimas.

As seen in Table 2, the libration amplitude of Mimas observed by Tajeddine et al.(2014) is $\gamma_{0}=50.3$ arc min. From equation (16) this implies an effective triaxiality $\sigma \approx 0.091$ provided that dissipation is weak. Together with the secular inward migration $\mathrm{da} / \mathrm{dt}=-15.7 \times 10^{-15}$ AU/day obtained by Lainey et al. (2012a) and other parameters of Table 2, this permit to deduce $\mathrm{Q}_{\mathrm{Pe}}$ from equation (20). One gets $\mathrm{Q}_{\mathrm{Pe}} \approx 26.8$. It may be seen a posteriori that the weak dissipation assumption $Q_{P e} \gg \sqrt{3 \sigma} /|3 \sigma-1|$ is fully satisfied.

From those values of $\sigma$ and $\mathrm{Q}_{\mathrm{Pe}}$, the expected phase shift of libration with respect to mean motion can be obtained from equation (15), yielding $\tan (\delta \theta) \approx 2.67 \times 10^{-2}$, or equivalently $\delta \theta \approx 1.53^{\circ}$ (modulo $180^{\circ}$ ).

Tajeddine et al. (2014) have measured the libration phase at J2000 and compared it to the theoretical phase computed by Noyelles et al. (2011) under the undamped hypothesis (see Table 2). The observed phase difference $\delta \theta=6.35^{\circ}$ should in principle account for dissipation. Surprisingly, this is much higher than the expected $\delta \theta \approx 1.53^{\circ}$ computed above, and the discrepancy between the expected and measured phase shift is much larger than the $\pm 0.8^{\circ}$ uncertainty of the measured phase quoted by the authors. In the low migration hypothesis discussed above (da/dt reduced by a factor of 10 from the one inferred by Lainey et al.(2012a)), one would expect $\delta \theta \approx 0.15^{\circ}$, and thus the discrepancy with the observed phase difference $\delta \theta=6.35^{\circ}$ would be even stronger.

This leads us to abandon the model with nonhydrostatic core and solid shell, and to explore a description similar to Tajeddine et al.'s (2014) alternative approach including an internal ocean.

\subsection{Model including inner core, internal ocean, and icy shell:}

An interior model of Mimas should be able to reproduce the different observations including the 3 main following features: secular inward motion $\mathrm{da} / \mathrm{dt}$, libration amplitude $\gamma_{\mathrm{o}}$, and libration phase shift $\delta \theta$. Here we describe Mimas as being composed of an inner core mostly composed of silicates, surrounded by a water ocean and an icy solid shell. Each of those three media is supposed to be homogeneous. The densities are labeled $\rho_{\mathrm{i}}, \rho_{\mathrm{O}}$, and $\rho_{\mathrm{S}}$ for the inner core, ocean, and shell, respectively. Due its uncertainty, the inner core density $\rho_{i}$ was varied from 1500 to $3300 \mathrm{~kg} / \mathrm{m}^{3}$ in the simulations, following Tajeddine et al. (2014). As concerns the water and ice densities $\rho_{O}$ and $\rho_{S}$, they were taken as $\rho_{O}=1000 \mathrm{~kg} / \mathrm{m}^{3}, \rho_{S}=900 \mathrm{~kg} / \mathrm{m}^{3}$ in the standard version of the model. Model runs were also performed with different ice and water densities, as discussed below. The outer boundaries of those three media are modeled as triaxial ellipsoids with semi-axes $c_{x}<b_{x}<a_{x}$, where subscript $x$ stands for $i, o$, or $s$ for the inner core, ocean, or shell, respectively. The equatorial flattening of each surface is $\beta_{x}=\left(a_{x}-b_{x}\right) / a_{x}$. In the context where the icy shell thickness is small, Richard et al. (2014) obtained that the 
flattening of the ocean is almost equal to the shell flattening. Assuming that this can be the case for Mimas, following those authors I take $\beta_{\mathrm{o}} \approx \beta_{\mathrm{S}}$. Note that for an homogeneous ellipsoid, the equatorial flattening $\beta$ is equal, to first order, to the triaxiality $\sigma$ introduced in section 2 . The dynamics of this composite body may be described by the following system, where the visco-elastic deformations of the shell and core have been ignored (Van Hoolst et al., 2008):

$$
\begin{aligned}
& C_{S} \ddot{\gamma}_{S}+\omega_{S}^{2} C_{S} \gamma_{S}+2 K\left(\gamma_{S}-\gamma_{i}\right)=2 e \omega_{S}^{2} C_{S} \sin n t \\
& C_{i} \ddot{\gamma}_{i}+\omega_{i}^{2} C_{i} \gamma_{i}-2 K\left(\gamma_{S}-\gamma_{i}\right)=2 e \omega_{i}^{2} C_{i} \sin n t
\end{aligned}
$$

where

$$
\begin{aligned}
& C_{S}=\frac{8 \pi}{15} \rho_{S}\left(R_{S}^{5}-\left(R_{S}-h\right)^{5}\right) ; \quad C_{i}=\frac{8 \pi}{15} \rho_{i} R_{i}^{5} \\
& \omega_{S}=n \sqrt{3 \beta_{S} \frac{\left(\rho_{O}-\rho_{S}\right)\left(R_{S}-h\right)^{5}+\rho_{S} R_{S}^{5}}{\rho_{S}\left(R_{S}^{5}-\left(R_{S}-h\right)^{5}\right)}} \\
& \omega_{i}=\sqrt[n]{3 \beta_{i} \frac{\rho_{i}-\rho_{O}}{\rho_{i}}} \\
& K=\frac{4 \pi}{5} G \rho_{O} \frac{\rho_{i}-\rho_{O}}{\rho_{i}} \beta_{S} \beta_{i} C_{i}
\end{aligned}
$$

In these expressions, $\gamma_{i}$ and $\gamma_{S}$ are physical librations of core and shell, respectively. $R_{i}$ and $R_{S}$ are the outer radii of core and shelt, and $\mathrm{h}$ is the shell thickness.

The damping of librations is introduced in a similar fashion as was done in section 2 (equation (9), where pendulum quality factors $\mathrm{Q}_{\mathrm{PeS}}$ and $\mathrm{Q}_{\mathrm{Pei}}$ are defined for the shell and core, respectively, according to equation (11). This gives:

$$
\begin{aligned}
& C_{S} \ddot{\gamma}_{S}+\omega_{S}^{2} C_{S} \gamma_{S}+2 K\left(\gamma_{S}-\gamma_{i}\right)+\frac{\omega_{S} C_{S}}{Q_{P e S}} \dot{\gamma}_{S}=2 e \omega_{S}^{2} C_{S} \sin n t \\
& C_{i} \ddot{\gamma}_{i}+\omega_{i}^{2} C_{i} \gamma_{i}-2 K\left(\gamma_{S}-\gamma_{i}\right)+\frac{\omega_{i} C_{i}}{Q_{P e i}} \dot{\gamma}_{i}=2 e \omega_{i}^{2} C_{i} \sin n t
\end{aligned}
$$

The question of dissipation within the ocean will be addressed below.

The solution of this linear system of forced damped coupled oscillators is obtained by substituting the physical librations of the core and shell $\gamma_{i}$ and $\gamma_{S}$ by their complex expressions:

$$
\bar{\gamma}_{S}=\bar{\gamma}_{S O} \exp (\text { int }) \quad ; \bar{\gamma}_{i}=\bar{\gamma}_{i O} \exp (\text { int })
$$


One obtains a linear system of 2 complex equations for $\bar{\gamma}_{S}$ and $\bar{\gamma}_{i}$ whose solutions are straightforward.

The pendulum quality factors of the core (presumably composed principally of silicates) and of the icy shell need not be the same, therefore several hypotheses have been tested from $\mathrm{Q}_{\mathrm{Pei}} / \mathrm{Q}_{\mathrm{PeS}}=0.1$ to $\mathrm{Q}_{\mathrm{Pei}} / \mathrm{QPeS}_{\mathrm{PeS}}=10$. Also, as mentioned above, different values of $\rho_{\mathrm{i}}$ were tested from $1500 \mathrm{~kg} / \mathrm{m}^{3}$ to $3300 \mathrm{~kg} / \mathrm{m}^{3}$. For each value of $\rho_{i}$ the core radius $R_{i}$ is deduced from the known mass and radius of Mimas. The 3 model parameters that are unknown are then: the shell thickness $h$, the inner core equatorial flattening $\beta_{\mathrm{i}}$, and the inner core pendulum quality factor $Q_{\text {Pei }}$. The whole parameter space of the input triplet $\left(\mathrm{h}, \beta_{\mathrm{i}}, \mathrm{Q}_{\mathrm{Pei}}\right)$ is explored, and each time the triplet $\left(\mathrm{da} / \mathrm{dt}, \gamma_{\mathrm{O}}, \delta \theta\right)$ is computed. This solution is then compared with the observed triplet $\left(\mathrm{da} / \mathrm{dt}=-15.7 \times 10^{-15} \mathrm{AU} / \mathrm{day}, \gamma_{\mathrm{O}}=50.3\right.$ arc min, $\left.\delta \theta=6.35^{\circ}\right)$. Provided that it exists, the input triplet that cancels the difference with the observed triplet is retained as the model solution.

Figure 2 displays the result for the icy shell thickness $\mathrm{h}$ as a function of the core density. One sees that the thickness is of the order of 16 to $35 \mathrm{~km}$ depending upon the assumed core density and $\mathrm{Q}_{\mathrm{Pei}} / \mathrm{Q}_{\mathrm{Pes}}$ ratio. This is of the same order as the thickness of $24 \mathrm{~km}$ determined by Tajeddine et al. (2014) in their viscoelastic shell assumption, or the thickness of $32 \mathrm{~km}$ that they obtain in their rigid shell assumption. However, this comparison has limited significance since, unlike Tajeddine et al.'s (2014) three-layer-model, the inner core here is allowed to have a non-hydrostatic shape.

Figure 3 displays the core equatorial flattening $\beta_{i}$ as a function of core density. The inferred value of $\beta_{\mathrm{i}}$ is weakly sensitive to the $\mathrm{Q}_{\mathrm{Pe}} / \mathrm{Q}_{\mathrm{PeS}}$ ratio, and ranges from 0.38 to 0.21 with increasing core density. This quite large value indicates that a non-hydrostatic core is indeed necessary to account for the observations. This feature is principally due to the high phase lag $\delta \theta \approx 6.35^{\circ}$ obtained by Tajeddine et al. (2014).

Figure 4 shows the pendulum quality factor $\mathrm{Q}_{\mathrm{Pei}}$ of the core, as a function of core density. $Q_{\text {Pei }}$ increases significantly for $\approx 80$ to $\approx 380$ for increasing core density. Its value is however virtually independent of the $\mathrm{Q}_{\mathrm{Pei}} / \mathrm{Q}_{\mathrm{Pes}}$ ratio, except for the largest value $\mathrm{Q}_{\mathrm{Pei}} / \mathrm{Q}_{\mathrm{PeS}}=10$ at low core density. In the absence of further information about the core density, one sees that there is a significant range of possible values for $\mathrm{Q}_{\mathrm{Pei}}$.

Once the three model parameters $h, \beta_{i}$ and $Q_{\text {Pei }}$ have been determined, one can deduce all the other quantities of the system. For example, Figure 5 shows the libration amplitude $\gamma_{\mathrm{iO}}$ of the inner core, as a function of core density. One sees that the core undergoes considerable libration, with amplitude varying from $\approx 2.5^{\circ}$ to $\approx 14^{\circ}$ for increasing core density if $\mathrm{Q}_{\mathrm{Pei}} / \mathrm{QPeS}_{\mathrm{P}} 1$. The amplitude of libration is seen to be nearly independent upon the $\mathrm{Q}_{\mathrm{Pei}} / \mathrm{Q}_{\mathrm{PeS}}$ ratio if $\mathrm{Q}_{\mathrm{Pei}} / \mathrm{Q}_{\mathrm{PeS}} \leq 1$ (dashed lines), but significantly dependent upon it if $\mathrm{Q}_{\mathrm{Pei}} / \mathrm{Q}_{\mathrm{PeS}} \geq 1$ (dotted lines).

The model was also run under the low migration hypothesis discussed above where da/dt is reduced by a factor of 10 from the one inferred by Lainey et al.(2012a)). The general behavior of the variations of the inferred parameters with inner core density (not shown) are very similar with the ones displayed in Figure 2 to Figure 5, although the range of values of the inferred parameters are modified. With this low da/dt approach, the inferred shell thickness $\mathrm{h}$ now ranges from $h \approx 28 \mathrm{~km}$ for core density $\rho_{\mathrm{i}}=1500 \mathrm{~kg} / \mathrm{m}^{3}$ to $\mathrm{h} \approx 21 \mathrm{~km}$ for $\rho_{\mathrm{i}}=3300 \mathrm{~kg} / \mathrm{m}^{3}$. 
Similarly the equatorial flattening $\beta_{\mathrm{i}}$ ranges from $\beta_{\mathrm{i}} \approx 0.36$ to $\beta_{\mathrm{i}} \approx 0.21$, and the core libration amplitude $\gamma_{\text {io }}$ ranges from $\gamma_{\mathrm{io}} \approx 2.1^{\circ}$ to $\gamma_{\mathrm{io}} \approx 11.1^{\circ}$. Those parameters are therefore moderately affected by the change of da/dt. On the contrary, the inferred $\mathrm{Q}_{\mathrm{Pei}}$ is strongly increased as compared with the one of Figure 4, ranging now from $\mathrm{Q}_{\mathrm{Pei}} \approx 500$ to $\mathrm{Q}_{\mathrm{Pei}} \approx 3300$.

From those results we conclude that among both interior models retained by Tajeddine et al. (2014) (solid body with nonhydrostatic core, or 3-layer body including ocean) only the latter is able to reproduce the combined set of three major observations: libration amplitude $\gamma_{\mathrm{O}}$, libration phase $\delta \theta$, and inward migration da/dt. Within the assumption that inward migration is mainly due to librational dissipation, this permits to determine $h, \beta_{\mathrm{i}}$ and $\mathrm{Q}_{\mathrm{Pei}}$ provided that an assumption is made concerning the core density. Due to the uncertainty of da/dt inferred from observations, the value of $Q_{P e i}$ is however rather uncertain. Note that unlike Tajeddine et al.'s (2014) three-layer model, a nonhydrostatic core is needed here in order to account for the observations.

\subsection{Dissipation within the ocean}

In the preceding section we have ignored the dissipation occurring within the ocean. The Reynolds number of the flow within the ocean is $\mathrm{Re} \approx \mathrm{VL} / \mathrm{v}$, where $\mathrm{V}$ is the azimuthal velocity of the shell with respect to the core, $\mathrm{L}$ is the ocean depth, and $v$ is the ocean kinematic viscosity. For the range of core densities explored in our model computations, the ocean depth is always larger than $5 \mathrm{~km}$, and the amplitude of the azimuthal deviation between core and shell is always larger than $2 \mathrm{~km}$. Assuming an ocean water viscosity of $10^{-6} \mathrm{~m}^{2} / \mathrm{s}$, one gets Re $>10^{8}$ which implies a turbulent boundary layer. Following Goldreich and Soter (1966), the rate of energy dissipation per unit area due to boundary layer turbulence may be estimated as $\left\langle-\dot{E}_{O}\right\rangle=f \rho_{O}<v^{3}>$, where $\mathrm{f} \approx 0.002$ is the coefficient of skin friction and $\mathrm{v}$ is the local velocity of the oceanic current. We perform the averaging of $\mathrm{v}^{3}$ over the spherical area and over time, and we get an estimate of the dissipated power by adding the powers dissipated in both the inner and outer boundary layers of the ocean. This gives:

$$
\left\langle-\dot{E}_{O}\right\rangle \approx \pi f \rho_{O} n^{3}\left[\gamma_{i O}^{3} R_{i}^{5}+\gamma_{S O}^{3} R_{O}^{5}\right]
$$

where $\gamma_{\mathrm{i}}$ and $\gamma_{\mathrm{SO}}$ are the libration amplitudes of core and shell, respectively, and $\mathrm{R}_{\mathrm{O}}$ is the outer radius of the ocean.

The result (not shown) is an increasing function of the assumed core density. In all cases we get $\left\langle-\dot{E}_{O}\right\rangle \leq 9 \times 10^{10}$ Watts, essentially due to dissipation at the core-ocean boundary layer. As a comparison, the dissipated power consistent with the observed inward migration of Mimas is:

$$
\langle-\dot{E}\rangle=\frac{-\dot{a}}{2 a^{2}} G m_{S} M_{P}=5.65 \times 10^{11} \text { Watts }
$$


Such level of energy produced by librational dissipation $\left(5.65 \times 10^{11}\right.$ Watts in equation (33)) is extremely high. It is two orders of magnitude higher than the observed heat flux at the South pole of Enceladus, and in the same order of magnitude as Europa. On would expect the surface of a satellite with the size of Mimas with such high energy production to look rather like Io. Since Mimas has almost no geological activity on its surface such high level of dissipation may seem questionable. It is therefore worthwhile to also explore the low migration hypothesis discussed above, in which da/dt is reduced by a factor of 10 . In that case, the dissipated power is reduced accordingly, yielding $\langle-\dot{E}\rangle=5.65 \times 10^{10}$ Watts from equation (33). As a comparison, dissipation in the ocean $\left\langle-\dot{E}_{O}\right\rangle$ obtained from equation (32) now ranges from $4.9 \times 10^{9}$ Watts to $4.4 \times 10^{10}$ Watts depending of core density from $1500 \mathrm{~kg} / \mathrm{m}^{3}$ to $3300 \mathrm{~kg} / \mathrm{m}^{3}$. Thus in that case, for high density core, dissipation within the ocean boundary layer $\left\langle-\dot{E}_{O}\right\rangle$ becomes the dominant cause for damping.

One concludes that dissipation within the ocean is expected to contribute significantly to the total dissipated power. Dissipation in the ocean has not been taken into account explicitly in the study performed above. The pendulum quality factors $\mathrm{Q}_{\mathrm{Pei}}$ and $\mathrm{Q}_{\mathrm{PeS}}$ that we inferred for core and shell may therefore be regarded as also including the energy dissipated in the inner and outer oceanic boundary layers, respectively.

\subsection{Other hypotheses for ice and ocean density:}

As seen above, the standard values of ice and water densities were taken as $\rho_{S}=900 \mathrm{~kg} / \mathrm{m}^{3}$ and $\rho_{\mathrm{O}}=1000 \mathrm{~kg} / \mathrm{m}^{3}$ in the model. However, ice density might be lower due to porosity, while ocean density might be higher due to salts. Other hypotheses were therefore also tested with modified shell or ocean densities, yielding somewhat modified results.

Thus, compared with the standard version, models with $\rho_{S}=700 \mathrm{~kg} / \mathrm{m}^{3}$ instead of $900 \mathrm{~kg} / \mathrm{m}^{3}$ were found compatible with the observed quantities, provided that $\mathrm{h}$ is increased by $80 \%$ to $30 \%$, and $\beta_{\mathrm{i}}$ and $\mathrm{Q}_{\mathrm{Pei}}$ are decreased by $24 \%$ to $3 \%$, and $56 \%$ to $47 \%$, respectively, depending on $\rho_{\mathrm{i}}$ from 1500 to $3300 \mathrm{~kg} / \mathrm{m}^{3}$.

Similarly models were tested with $\rho_{\mathrm{O}}>1000 \mathrm{~kg} / \mathrm{m}^{3}$. Incidentally, for $\rho_{\mathrm{O}}$ close to $1100 \mathrm{~kg} / \mathrm{m} 3$ (with $\rho_{\mathrm{S}}=900 \mathrm{~kg} / \mathrm{m}^{3}$ and $\rho_{\mathrm{i}}=3300 \mathrm{~kg} / \mathrm{m}^{3}$ ), the triplet $\left(\mathrm{h}, \beta_{\mathrm{i}}, \mathrm{Q}_{\mathrm{Pei}}\right.$ ) required to account for the set of observations approaches a resonant situation, yielding a core libration amplitude $\gamma_{\mathrm{iO}}$ as large as $\approx 35^{\circ}$. For such specific resonance situation ocean dissipation as given by equation (32) would become comparable to the $5.65 \times 10^{11}$ Watts given by equation (33). In that case, dissipation in the ocean would be sufficient to account by itself for the whole libration damping. One may not exclude the possibility that such resonant situations would have occurred incidentally during limited periods in the past.

\section{Conclusion and discussion}

The strong inward migration da/dt obtained by Lainey et al. (2012a) for Mimas is an intriguing feature that has not yet received convincing explanation. Mimas is a triaxial moon on an eccentric orbit, and as a consequence it is librating. Libration is believed to enhance significantly dissipation within the moon (Makarov and Efroimsky; 2014; Makarov et al., 
2016). Mimas' inward migration is interpreted here as an effect of dissipation within the librating moon. Tajeddine et al. (2014) performed observations of the phase and amplitude of libration and proposed several interior models, from which they retained only two models compatible with observations: a solid body with nonhydrostatic core, or 3-layer body including an inner dense core, surrounded by a water ocean and an icy solid shell. In this paper I combined three major observations: libration amplitude $\gamma_{O}$ and libration phase $\delta \theta$ observed by Tajeddine et al. (2014), and inward migration da/dt obtained by Lainey et al. (2012a). A further study by Lainey et al. (2015) however tends to indicate that the Mimas' strong inward motion might be questioned, and therefore this paper also explores situations with smaller da/dt. Within the assumption that inward migration is mainly due to librational dissipation, the solid model with nonhydrostatic core is found to be inconsistent with the observations. In contrast, a 3-layer model including core, ocean and shell is compatible. The observations permit to determine the icy shell depth $h$, core equatorial flattening $\beta_{i}$ and core pendulum quality factor $\mathrm{Q}_{\mathrm{Pei}}$, provided that an assumption is made concerning the core density (the visco-elastic deformation of core and shell are ignored). Depending upon the assumed core density between 1500 and $3300 \mathrm{~kg} / \mathrm{m}^{3}$ and upon the $Q_{\mathrm{Pei}} / \mathrm{Q}_{\mathrm{PeS}}$ ratio from 0.1 to 10 , the range of values are $[35 \mathrm{~km}-16 \mathrm{~km}],[0.38-0.21]$, respectively for $\mathrm{h}$ and $\beta_{\mathrm{i}}$. Due to the uncertainty of da/dt inferred from observations, the value of $Q_{P e i}$ is however rather uncertain. Note that unlike Tajeddine et al.'s (2014) three-layer model, the core here needs to be nonhydrostatic in order to account for the observations.

Due to conservation of momentum the inward migration of Mimas should be accompanied with a decrease of orbital eccentricity. One may thus expect that after a while the eccentricity would be reduced and that the effect of libration on da/dt would eventually vanish if no other process acts to maintain eccentricity. This inward migration of Mimas is therefore not expected to be a permanent feature. In the absence of a forcing source, if only dissipation within the satellite is considered, the decay of eccentricity is given by (Murray and Dermott, 1999, page 167):

$$
\dot{e} \approx-\frac{\dot{E}}{2 e E}
$$

Since $\mathrm{E}$ is inversely proportional to a, this implies $\dot{e} \approx \dot{a} /(2 e a)$, and thus in the context of librational dissipation the eccentricity damping timescale of Mimas can be written as:

$$
\tau_{e}=-\frac{e}{\dot{e}} \approx-\frac{2 a}{\dot{a}} e^{2}
$$

Lainey et al. (2012b) have suggested that an orbital inward migration of Mimas could produce a division in the rings similar to the observed Cassini division. Taking $\dot{a}=-15.7 \times 10^{-15} \mathrm{AU} / \mathrm{day}$ from Lainey et al. (2012a), equation (35) would yield $\tau_{\mathrm{e}} \approx 0.18$ Myear, while the time required for Mimas to cover the radial extent of distance Cassini's division (about $4830 \mathrm{~km}$ ) would be $\approx 5.6$ Myears. Thus, assuming that Lainey et al.'s(2012b) scenario took place temporarily in 
the past due to enhanced dissipation within Mimas, some process should have been acting during that period to maintain eccentricity.

Acknowledgements

The author thanks Michael Efroimsky and another reviewer for their constructive comments, which permitted to improve considerably the quality of the paper.

Table 1. Physical and orbital properties of Phobos and Mars

\begin{tabular}{lll}
\hline Parameter & value & reference \\
\hline Mars: & & \\
Mass $\mathrm{M}_{\mathrm{P}}$ & $6.42 \times 10^{23} \mathrm{~kg}$ & Konoplivet al. (2006) \\
Spin period $\mathrm{T}_{\mathrm{P}}=2 \pi / \Omega_{\mathrm{P}}$ & 24.623 hours & Murray and Dermott (1999) \\
& & \\
Phobos: & $1.06 \times 10^{16} \mathrm{~kg}$ & Andert et al. (2010) \\
Mass $\mathrm{m}_{\mathrm{S}}$ & $13 \times 11.39 \times 9.07 \mathrm{~km}$ & Willner et al. (2010) \\
Radii & $11.27 \mathrm{~km}$ & quadratic mean of radii \\
Mean radius $\mathrm{R}_{\mathrm{S}}$ & $0.3362,0.3871,0.4773$ & Willner et al. (2010) \\
Normalized MOI $(\mathrm{A}, \mathrm{B}, \mathrm{C})$ & 0.129 & O=(B-A)/C \\
Shape based triaxiality & 0.01511 & Jacobson $(2010)$ \\
Orbit eccentricity e & $9,375 \mathrm{~km}$ & Jacobson $(2010)$ \\
Orbit semi-major axis a & $2.28 \times 10^{-4} \mathrm{rad} / \mathrm{s}$ & n=(GM $\left./ \mathrm{a}^{3}\right)^{1 / 2}$ \\
Mean motion $\mathrm{n}$ & $1.27 \times 10^{-3} \mathrm{deg} / \mathrm{yr}{ }^{2}$ & Lainey et al. (2007) \\
Secular acceleration $1 / 2 \mathrm{dn} / \mathrm{dt}$ & $1.2^{\circ}$ & Willner et al. (2010) \\
Libration amplitude & & \\
\hline
\end{tabular}

* MOI= moment of inertia 
Table 2. Physical and orbital properties of Mimas and Saturn

\begin{tabular}{|c|c|c|}
\hline Parameter & value & reference \\
\hline \multicolumn{3}{|l|}{ Saturn: } \\
\hline Mass $M_{P}$ & $5.6846 \times 10^{26} \mathrm{~kg}$ & Murray and Dermott (1999) \\
\hline Radius $R_{P}$ & $60,330 \mathrm{~km}$ & Murray and Dermott (1999) \\
\hline Spin period $T_{P}=2 \pi / \Omega_{P}$ & 10.656 hours & Murray and Dermott (1999) \\
\hline Dissipation ratio $\mathrm{k}_{2 \mathrm{P}} / \mathrm{Q}_{\mathrm{P}}$ & $2.3 \times 10^{-4}$ & Lainey et al. (2012a) \\
\hline \multicolumn{3}{|l|}{ Mimas: } \\
\hline Mass $\mathrm{m}_{\mathrm{S}}$ & $3.7493 \times 10^{19} \mathrm{~kg}$ & Jacobson et al.(2006) \\
\hline Radii & $207.8 \times 196.7 \times 190.6 \mathrm{~km}$ & Thomas (2010) \\
\hline Mean radius $R_{S}$ & $198.5 \mathrm{~km}$ & quadratic mean of radii \\
\hline Shape based triaxiality $\sigma$ & 0.06 & ad Thomas(1988) \\
\hline Libration amplitude (measured) & $50.3 \pm 1.0$ & Tajeddine et al. (2014) \\
\hline Libration amplitude (theoretical) & 26.07 arc & Noyelles et al. (2011) \\
\hline Librat. phase at J2000(measured) & $107.7 \pm 0.8^{\circ}$ & Tajeddine et al. (2014) \\
\hline Librat. phase at J2000(theoretical) & $101.35^{\circ}$ & Noyelles et al. (2011) \\
\hline Orbit eccentricity e & 0.0202 & Murray and Dermott(1999) \\
\hline Orbit semi-major axis a & $185,520 \mathrm{~km}$ & Murray and Dermott (1999) \\
\hline Secular da/dt & $-15.7 \times 10^{-15}$ AU/day $\left(^{*}\right)$ & Lainey et al. (2012a) \\
\hline Orbital period $\mathrm{T}$ & & Murray and Dermott (1999) \\
\hline Mean motion $\mathrm{n}$ & & $\mathrm{n}=2 \pi / \mathrm{T}$ \\
\hline
\end{tabular}

(*) value later on questioned by Lainey et al. (2015)

\section{REFERENCES}

Andert, T. P., Rosenblatt, P., Pätzold, M., Häusler, B., Dehant, V., Tyler, G. L., and Marty, J. C., 2010. Precise mass determination and the nature of Phobos. Geophys. Res. Lett., 37, L09202 (4pp.).

Bills, B. G., Neumann, G. A., Smith, D. E., and Zuber, M. T., 2005. Improved estimate of tidal dissipation within Mars from MOLA observations of the shadow of Phobos. J. Geophys. Res., 110, E07004 (15pp.).

Caudal, G. V., 2013. The role of tidal torques on the evolution of the system of Saturn's coorbital satellites Janus and Epimetheus, Icarus, 223, 733-740. 
Comstock, R. L., and B. G. Bills, 2003. A solar system survey of forced librations in longitude, J. Geophys. Res., 108, E9, 5100, JE002100.

Dermott, S. F., and Thomas, P. C., 1988. The shape and internal structure of Mimas, Icarus, $73,25-65$.

Efroimsky, M., and Makarov, V. V., 2014. Tidal dissipation in a homogeneous spherical body. I. Methods. The Astrophysical Journal, 795:6 (19pp).

Goldreich, P., and Soter, S., 1966. Q in the solar system. Icarus, 5, 375-389.

Goldreich, P., and Tremaine, S., 1979. The excitation of density waves at the Lindblad and corotation resonances by an external potential. The Astrophysical Journal, 233: 857-871.

Goldreich, P., and Tremaine, S., 1980. Disk-satellite interactions. The Astrophysical Journal, 241:425-441.

Jacobson, R. A., 2010. The orbits and masses of the Martian satellites and the libration of Phobos. The Astronomical Journal, 139:668-679.

Jacobson, R. A., Antreasian, P. G., Bordi, J. J., Criddle, K. E., Ionasescu, R., Jones, J. B., Mackenzie, R. A., Meek, M. C., Parcher, D., Pelletier, F. J., Owen, W. M. Jr., Roth, D. C., Roundhill, I. M., and Stauch, J. R., 2006. The gravity field of the saturnian system from satellite observations and spacecraft tracking data, The Astronomical Journal, 132: 25202526.

Konopliv, A. S., Yoder, C. F., Myles Standish, E., Yuan, D.-N., Sjogren, W. L., 2006. A global solution for the Mars static and seasonal gravity, Mars orientation, Phobos and Deimos masses, and Mars Ephemeris. Icarus, 182, 23-50.

Lainey, V., Dehant, V., and Pätzold, M., 2007. First numerical ephemerides of the Martian moons. Astronomy and Astrophysics, 465, 1075-1084.

Lainey, V., Karatekin, O., Desmars, J., Charnoz, S., Arlott, J. E., Emelyanov, N., Le PoncinLafitte, C., Mathis, S., Remus, F., Tobie, G., and Zahn, J. P., 2012a. Strong tidal dissipation in Saturn and constraints on Enceladus' thermal state from astrometry. The Astrophysical Journal, 752:14 (19pp.).

Lainey, V., Charnoz, S., Reboussin, L., Noyelles, B., Baillié, K., 2012b. The Cassini Division and Mimas' eccentricity: A common history? AAS Div. Planet. Sci. Meet. 44, 414.08 (poster).

Lainey, V., Jacobson, R. A., Tajeddine, R., Cooper, N. J., Murray, C., Robert, V., Tobie, G., Guillot, T., Mathis, S., Remus, F., Desmars, J., Arlot, J.-E., De Cuyper, J.-P., Dehant, V., Pascu, D., Thuillot, W., Le Poncin-Laffite, C., Zahn, J.-P., 2015. New constraints on Saturn's interior from Cassini astrometric data. Earth and Planetary Astrophysics, arXiv:1510.05870 [astro-ph.EP]. 
Le Maistre, S., Rosenblatt, P., Rambaux, N., Castillo-Rogez, J. C., Dehant, V., and Marty, J. C., 2013. Phobos interior from librations determination using Doppler and star tracker measurements. Planetary and Space Science, 85, 106-122.

Makarov, V. V., Frouard, J., and Dorland, B., 2016. Forced libration of tidally synchronized planets and moons. Mon. Not. R. Astron. Soc., Vol. 456, 665-671.

Makarov, V. V., and Efroimsky, M., 2014. Tidal dissipation in a homogeneous spherical body. II. Three examples: Mercury, Io, and Kepler-10 b. The Astrophysical Journal, 795:7 (10pp.).

Molnar, L. A., and Dunn, D. E., 1995. The Mimas 2:1 eccentric corotational resonance in Saturn's outer B ring, Icarus, 116, 397-408.

Murray, C. D., and Dermott, S. F., 1999. Solar system dynamics, Cambridge University press, Cambridge.

Noyelles, B., Karatekin, O., and Rambaux, N., 2011. The rotation of Mimas. Astronomy and Astrophysics, 536, A61 (13pp.).

Ostrovsky, L. A., and Johnson, P. A., 2001. Dynamic nonlinear elasticity in geomaterials. Rivista del Nuovo Cimento, 24, 7, 1-46.

Peale, S. J., and Cassen, P., 1978. Contribution of tidal dissipation to lunar thermal history. Icarus, 36, 245-269.

Richard, A., Rambaux, N., and Charnay, B., 2014. Librational response of a deformed 3-layer Titan perturbed by non-Keplerian orbit and atmospheric couplings. Planetary and Space Science, 93-94, 22-34.

Tajeddine, R., Rambaux, N., Lainey, V., Charnoz, S., Richard, A., Rivoldini, A., and Noyelles, B., 2014. Constraints on Mimas' interior from Cassini ISS libration measurements. Science, 346, 322-324

Thomas, P. C., 2010. Sizes, shapes, and derived properties of the saturnian satellites after the Cassini nominal mission, Icarus, 208, 395-401.

Tiscareno, M. S. Thomas, P. C., and Burns, J. A., 2009. The rotation of Janus and Epimetheus, Icarus, 204, 254-261.

Van Hoolst, T., Rambaux, N., Karatekin, O., Dehant, V., and Rivoldini, A., 2008. The librations, shape, and icy shell of Europa. Icarus, 195, 386-399.

Willner, K., Oberst, J., Hussmann, H., Giese, B., Hoffmann, H., Matz, K. D., Roatch, T., and Duxbury, T., 2010. Phobos control point network, rotation and shape. Earth and Planetary Science Letters, 294, 541-546.

Wisdom, J., Peale, S. J., and Mignard, F., 1984. The chaotic rotation of Hyperion. Icarus 58, 137-152. 
Yoder, C. F., and Peale, S. J., 1981. The tides of Io. Icarus, 47, 1-35.

Yoder, C. F., 1982. Tidal rigidity of Phobos. Icarus, 49, 327-346.

Figure 1.

Representation of the angles used in the paper in a satellite centered frame rotating with the satellite's mean motion $\mathrm{n}$ : physical libration $\gamma$, optical libration $\psi$, and angle $\varphi=2 \operatorname{esin}(\mathrm{nt})$.

Figure 2.

Shell thickness $\mathrm{h}$ consistent with the observations, as a function of assumed core density. Solid line: $\mathrm{Q}_{\mathrm{Pei}} / \mathrm{Q}_{\mathrm{PeS}}=1$ assumed; dashed lines: $\mathrm{Q}_{\mathrm{Pei}} / \mathrm{Q}_{\mathrm{PeS}}=0.1$ or 0.33 assumed; dotted lines: $\mathrm{Q}_{\mathrm{Pei}} / \mathrm{Q}_{\mathrm{PeS}}=3$. or 10 . assumed.

Figure 3.

Same as Figure 2, but core equatorial flattening $\beta_{\mathrm{i}}$ is displayed.

Figure 4.

Same as Figure 2, but librational quality factor $\mathrm{Q}_{\text {Pei }}$ is displayed.

Figure 5.

Same as Figure 2, but the amplitude $\gamma_{\mathrm{iO}}$ of the inner core libration is displayed.

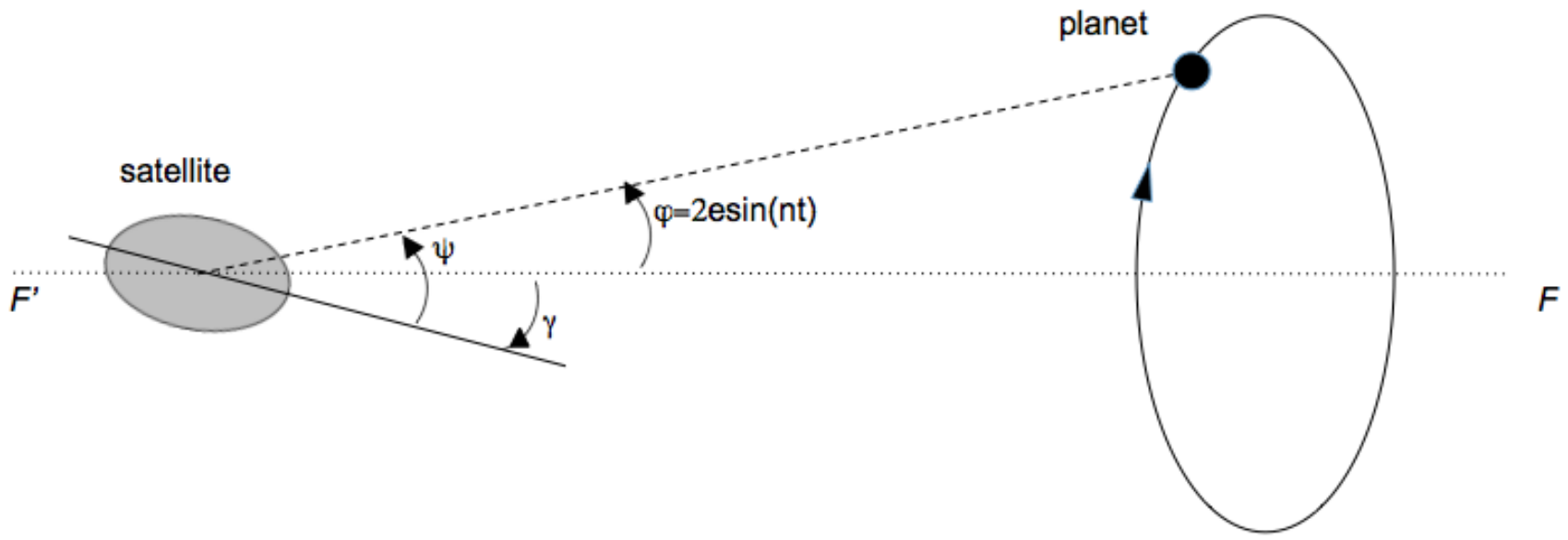

Figure 1.

Representation of the angles used in the paper in a satellite centered frame rotating with the satellite's mean motion n: physical libration $\gamma$, optical libration $\psi$, and angle $\varphi=2$ esin(nt). 


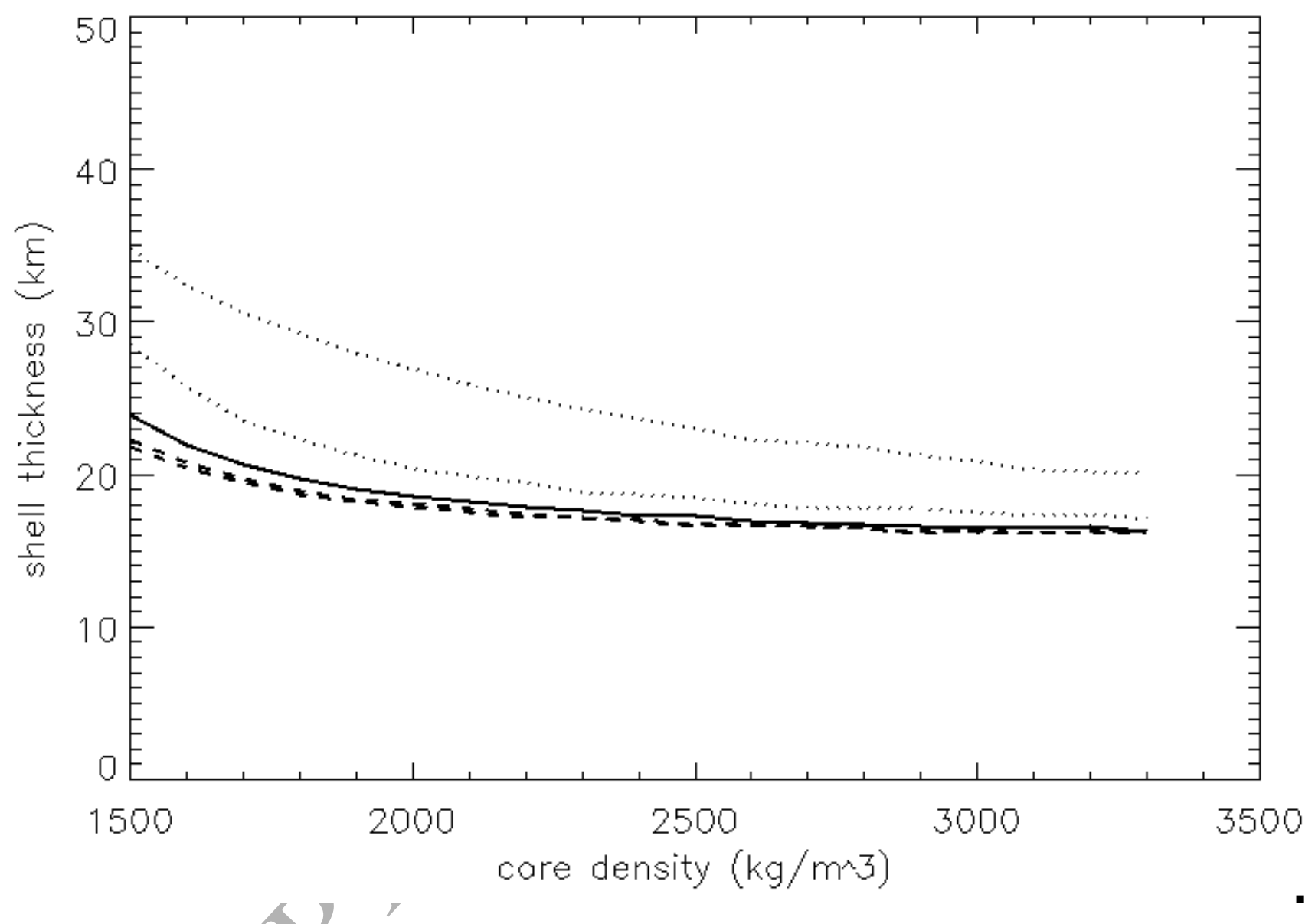

Figure 2.

Shell thickness $\mathrm{h}$ consistent with the observations, as a function of assumed core density. Solid line: $\mathrm{Q}_{\mathrm{Pei}} / \mathrm{Q}_{\mathrm{PeS}}=1$ assumed; dashed lines: $\mathrm{Q}_{\mathrm{Pei}} / \mathrm{Q}_{\mathrm{PeS}}=0.1$ or 0.33 assumed; dotted lines: $\mathrm{Q}_{\mathrm{Pei}} / \mathrm{Q}_{\mathrm{PeS}}=3$. or 10 . assumed. 


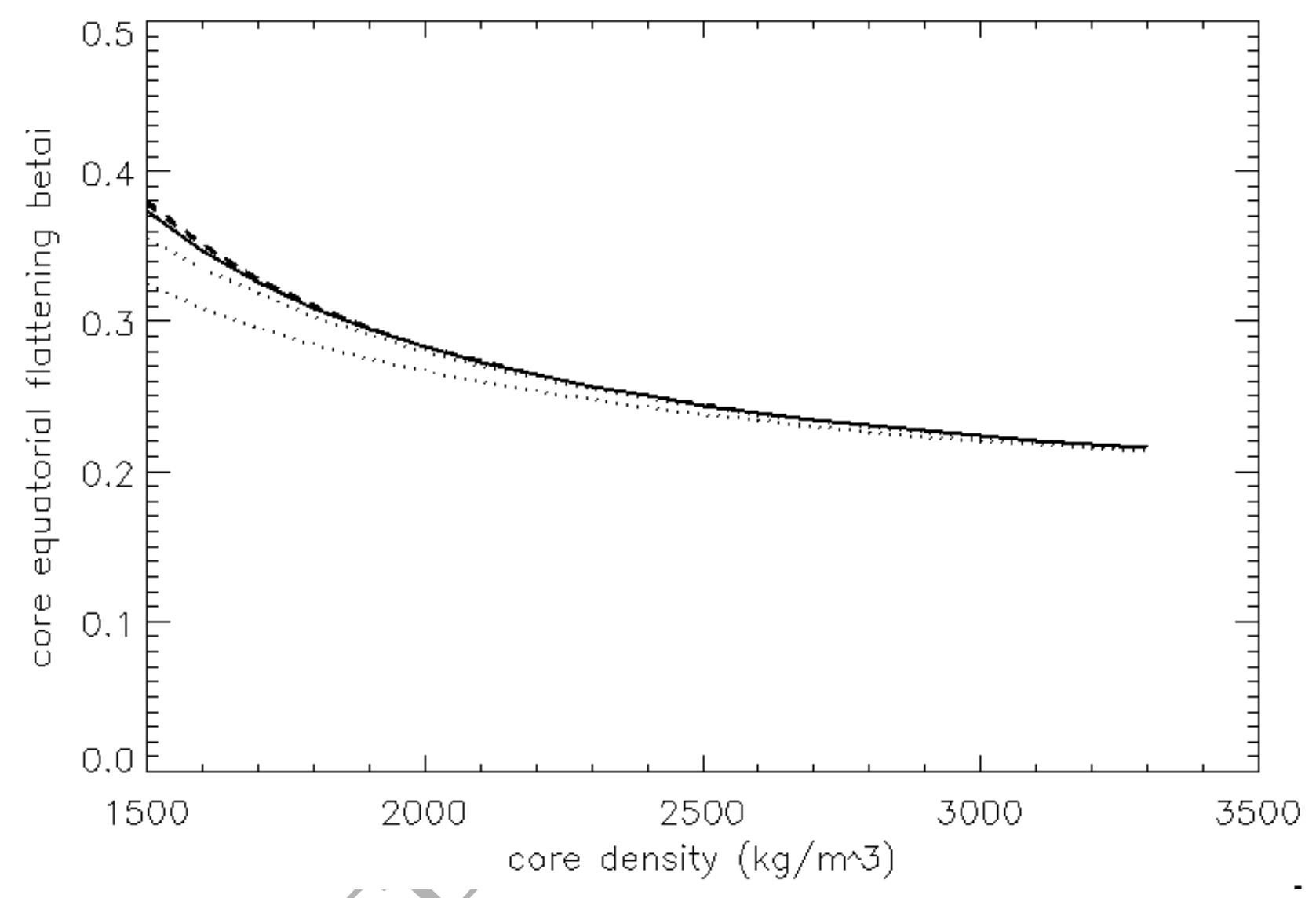

Figure 3.

Same as Figure 2, but core equatorial flattening $\beta_{\mathrm{i}}$ is displayed. 


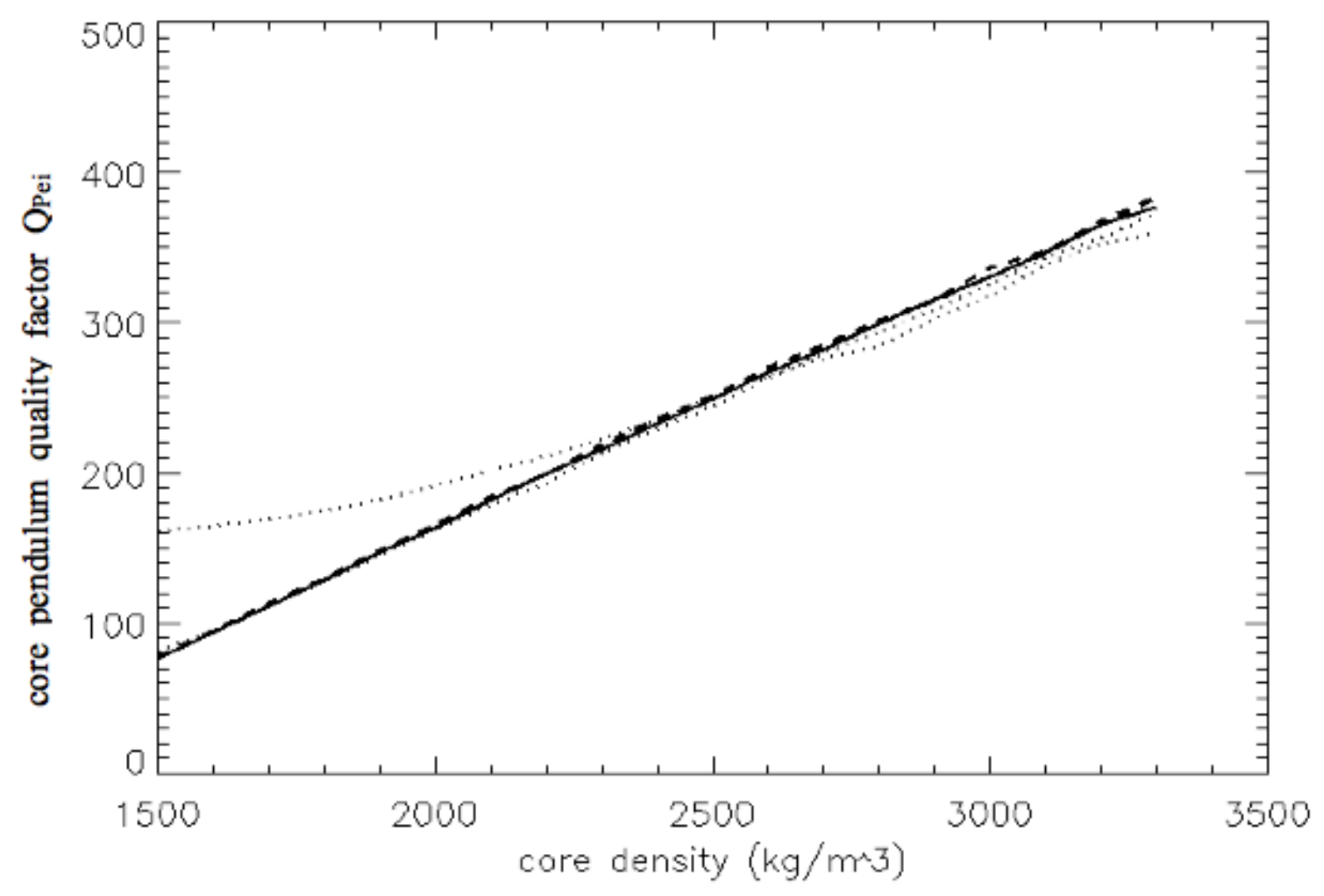

Figure 4.

Same as Figure 2, but librational quality factor $\mathrm{Q}_{\text {Pei }}$ is displayed. 


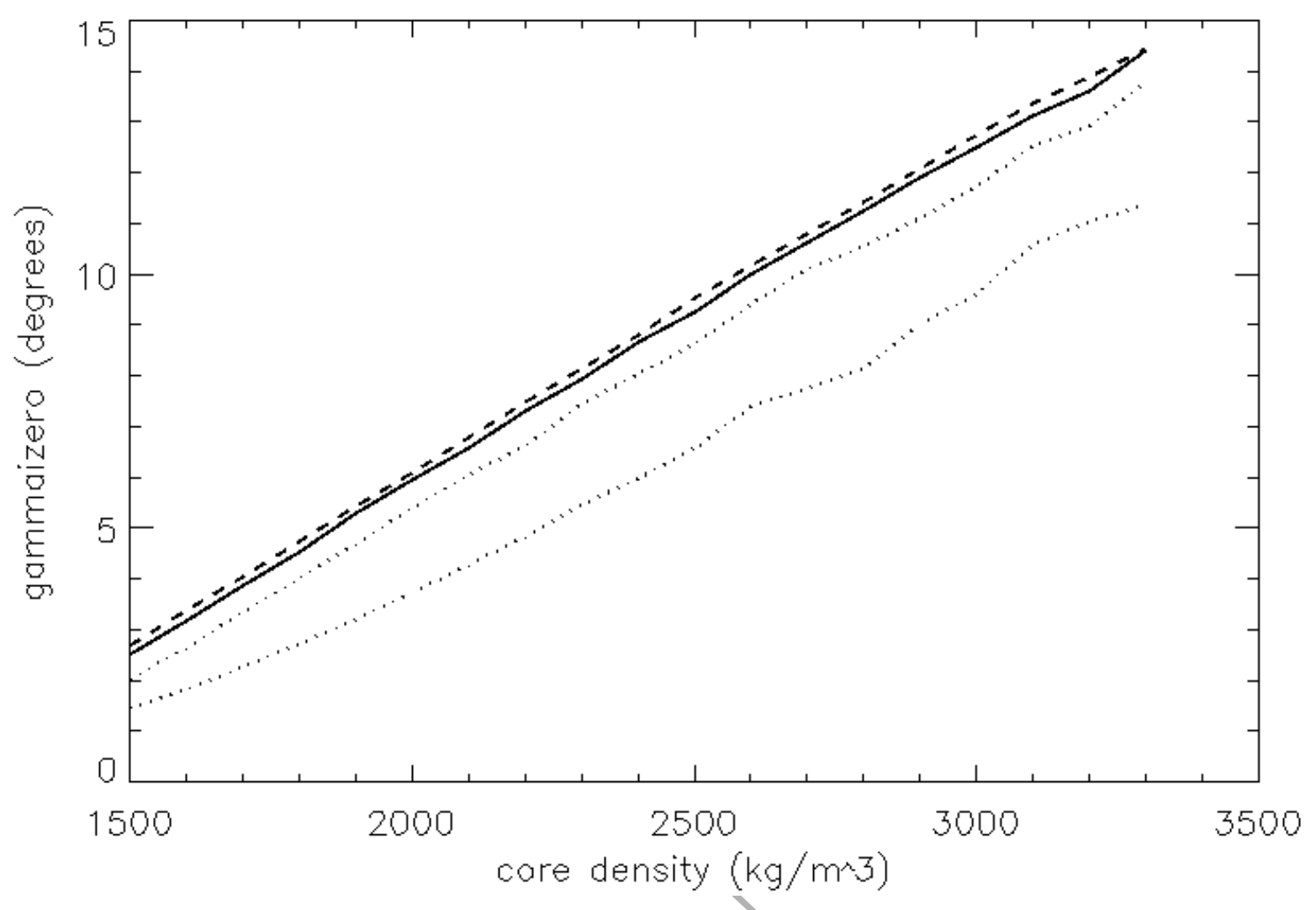

Figure 5.

Same as Figure 2, but the amplitude $\gamma_{\mathrm{iO}}$ of the inner core libration is displayed. 